\title{
Japanese Are Modest Even When They Are Winners: Competence Ratings of Winners and Losers in Social Comparison
}

\author{
Kazuo Mori ${ }^{1}$, Hideko Mori $^{2}$ \\ ${ }^{1}$ Institute of Engeneering, Tokyo University of Agriculture and Technology, Koganei, Tokyo, Japan \\ ${ }^{2}$ Bunka Gakuen Nagano College, Nagano, Japan \\ Email: kaz-mori@cc.tuat.ac.jp
}

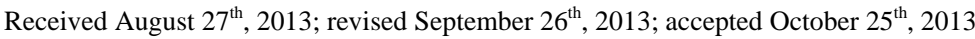

Copyright (C) 2013 Kazuo Mori, Hideko Mori. This is an open access article distributed under the Creative Commons Attribution License, which permits unrestricted use, distribution, and reproduction in any medium, provided the original work is properly cited.

\begin{abstract}
Social comparison experiments in two different social conditions, competing between friends and between strangers, were carried out with 88 Japanese male undergraduates. Participants were asked to come to the laboratory in friend pairs to participate in the experiment. Two pairs were randomly combined for each experimental session. In the Between-Friends condition, one of the two pairs solved 20 anagrams competitively while the other pair observed them. In the Between-Strangers condition, one performer and one observer were randomly chosen in each pair and the performers solved anagram tasks competitively. As in our previous study, the anagram tasks were presented utilizing a presentation trick so that one performer-and-observer group viewed easier anagrams than the other group without their noticing the difference. As intended, those who viewed the easier anagrams outperformed the others, becoming winners in all sessions. No participants noticed the trick. After the task, all four participants rated the ability of the two performers including themselves. Their ability ratings showed that they tended to evaluate their own ability modestly. Even winners consistently rated themselves lower than the others rated them. Two possible explanations of why Japanese participants made such modest responses were presented and discussed.
\end{abstract}

Keywords: Social Comparisons; Winners and Losers; Japanese Males; Self-Rating; fMORI Technique

\section{Introduction}

People may sometimes win and other times lose in various social comparisons. Losers may suffer damage to their confidence or self-esteem. Alicke, LoSchiavo, Zerbst, and Zhang (1997) assumed that a loser would be likely to exalt a winner excessively to protect his/her own confidence or self-esteem. By elevating the ability of the winners, rather than diminishing their own ability, losers can maintain their own sense of competence while magnanimously acknowledging the superior attributes of the winners. Alicke et al. (1997) tested this hypothesis by conducting an experiment with triads of participants (one being a confederate) in which a participant and the confederate competed on a kind of intelligence quiz while the other participant observed it. The participants were either to win or lose the competition regardless of their actual competency, because the confederates either deliberately outperformed them or let themselves be outperformed. After the competition, both participants (performers and observers) evaluated the intellectual ability of the counterparts (confederates). As hypothesized, those participants who had lost the competition tended to rate the ability of the winners much higher than the observing participants rated the winners. Alicke et al. dubbed this loser's tendency to exaggerate a winner's ability the "Genius Effect." The Genius Effect was repeatedly observed in follow-up studies (e.g., Lassiter \& Munhall, 2001). Lassiter, Clark, Munhall, and Lindberg (2008) extended this phenomenon to situations in which participants believed themselves to be not-so-competent beforehand, yet they still outperformed others. The researchers found that those participants tended to rate their contestants even lower than uninvolved observers rated them (the "Idiot Effect”). According to these studies, there seems a simple tendency: losers rate their opponents' ability higher and winners rate their opponents' ability lower than observers' neutral ratings.

In Alicke et al.'s study and all of the follow-ups, the participants were strangers to the confederates. Social comparison effects between two acquaintances are likely to be different from those between strangers. However, it is difficult to examine a social comparison between two acquaintances using the Alicke type experimental procedure because participants and confederates are basically strangers in those experiments.

Mori and Mori (2011) examined social comparison effects with groups of mutually acquainted participants rather than strangers. However, they replicated Alicke et al.'s (1997) study without using confederates. Instead, they utilized a presentation trick (fMORI Technique, Mori, 2007) to present easy and difficult anagram tasks on the same screen to a pair of genuine participants. Using special pairs of sunglasses polarized perpendicularly from each other, the two different sets of anagram tasks were presented to participants such that each pair was able to observe only one set or the other, depending on how their sunglasses were polarized. In this way, they successfully and artificially created winners and losers among genuine participant pairs because those who were given the easier tasks always out- 
performed the others.

However, the results of Mori and Mori (2011) were different from those of Alicke et al. (1997) and Lassiter et al. (2008). The losers in Mori and Mori (2011) rated the winner's intelligence equally, as did the observers. Specifically, they did not exaggerate their opponents' ability in order to protect their own self-esteem. Instead, they rated their own intelligence as lower than did the observers. Meanwhile, the interaction of those winners' ratings was also contrary to that reported in Lassiter et al. (2008). The winners in Mori and Mori (2011) rated themselves much lower than the observers and losers did, and rated the losers in the same way as the observers. Namely, neither Genius nor Idiot Effects were observed. Instead, both the winners and losers rated themselves lower than the others did. They dubbed it the "Modesty Effect."

The differences between the two studies, Alicke et al. (1997) and Mori and Mori (2011), might be attributable to two factors: i) cross-cultural differences between the participants, Americans and Japanese, or ii) the interpersonal relationships among the participants, strangers and acquaintances, in Alicke et al. (1997) and Mori and Mori (2011), respectively. It would be desirable to examine these variables by conducting an experiment with a two-factorial between-subject design. However, a cross-cultural experiment is not easy to carry out in Japan. Therefore, the present study aimed to examine only the second variable, strangers vs. friends, using all Japanese participants.

\section{Method}

\section{Participants}

Eighty-eight Japanese male undergraduates (18 - 23 years old, 20.0 years old on average) participated. As in Alicke et al. (1997), only male students were recruited for the study. The experimenters asked them to come to the laboratory in mutually acquainted pairs. Two not-acquainted pairs were randomly combined and regrouped into different arrangements according to the two experimental conditions (22 pairs to form 11 foursomes in each condition). In the Between-Friends condition, one of two pairs was randomly assigned to be Performers, who solved anagram tasks competitively, while the other pair were assigned to act as Observers. In the Between-Strangers condition, one Performer and one Observer were randomly chosen in each pair and those two Performers solved the tasks competitively.

\section{Procedure}

Random role assignment. Two participant pairs were allocated to the same experimental time slot. On arrival, they were assigned to one of the following roles: Performer of easy tasks (PE), Performer of difficult tasks (PD), and Observers (OE \& $\mathrm{OD})$. In the Between-Friends condition, the PE and PD were chosen from one of the pairs, while in the Between-Strangers condition, the PE and PD were chosen from each pair.

Anagram task instructions. The two pairs of participants were seated in two rows of two chairs each placed side-by-side approximately $1 \mathrm{~m}$ in front of the rear screen (see Figure 1). The front seats were for the Performers. The sunglasses suitable for each role were placed on each seat. Participants were told to wear the sunglasses to avoid glare. The participants were told that they would be given several word puzzles presented oneby-one on the screen in front of them. The Performers' task was to figure out the correct word by re-arranging the letter string shown to them, and announce the answer as soon as possible. The Observers sitting right behind the Performers were told that their task was to observe the activities of the two Performers and make a written record. Then, the Observers were given the check sheet to mark the record for each task.

Anagram task presentation. The same sets of anagram tasks as those in Mori and Mori (2011) were used in the present study. Easy and difficult anagram tasks were projected onto the same half-transparent screen utilizing the fMORI Technique (Mori, 2007) so that two participants (PE and OE) viewed easy ones while the other two (PD and OD) saw difficult ones. Each anagram task was presented for 20 seconds or until one of the Performers answered correctly, whichever came first, and the experimenter then proceeded to the next task. There were 24 anagram tasks with two practice items preceding them. It was designed such that the Easy task Performers (PEs) would answer about half of the test items correctly and so that the Difficult task Performers (PDs) might accidentally figure out the correct answers before their counterparts only once or twice during the whole session. It took about ten minutes to complete the anagram task session.

Intelligence ratings. After the anagram task session, participants were asked to rate their intellectual levels in a questionnaire format. The Performers rated the winners and losers, including themselves, while the Observers rated the winners and losers only. Ratings were made on a 10 -point scale ranging from 0 (extremely low) to 9 (extremely high). To attenuate any hesitation to rate other people's intelligence directly, the crucial rating was intermingled among a variety of filler questions addressing their attitudes, motivation, and other personality traits. It took about 15 minutes to complete the questionnaire.

Post-experimental interviews. After the questionnaire session, the experimenter asked first whether the participants had noticed any abnormality in the presentation or not. Then, he informed them about the research goals in order to obtain the informed consent of the participants. They were paid 1000 yen (about US \$10) each for their participation.

\section{Results}

\section{Effectiveness of the Experimental Manipulation}

Post-experimental interviews revealed that no participants had noticed the presentation trick. The easy-task-performers (PEs) outperformed their counterparts (PDs) in all 22 groups. The average numbers of correct responses of the PEs were 10.3 and 9.5 out of 24 tasks, for the Between-Friends condition and the Between-Strangers condition, respectively, while those of the PDs were 2.9 and 3.2, respectively. Therefore, the present experimental setting did seem to have successfully created winners and losers without utilizing confederates.

\section{Intelligence Ratings}

Figure 2 shows the means for the ratings of participants' intelligence. The main statistical analysis included two betweensubject variables, Social condition (Friends-Strangers) and Rater roles (Winners-Losers-Observers-Observers), and one within-subject variable, Rated roles (Winners-Losers).

A three-way mixed ANOVA revealed that the main effects of Social conditions (Friends-Strangers) and Rated roles (Winners-Losers) were significant. As expected, the winners' intelligence was rated statistically higher than that of the losers 


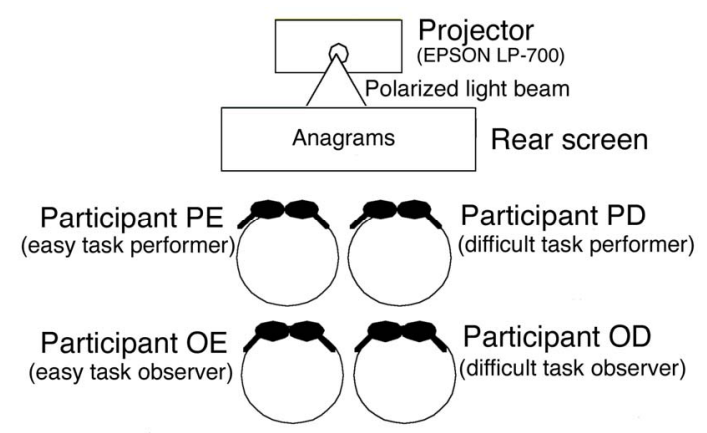

Figure 1.

Diagram of the experimental setting.

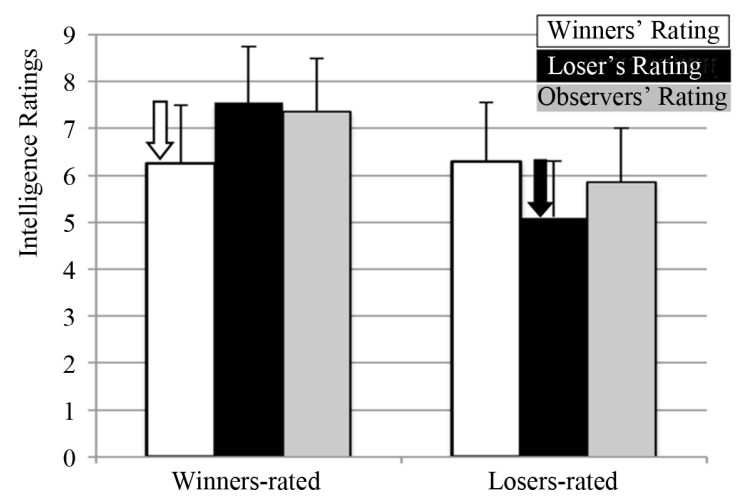

Figure 2.

Winners and Losers rated for their intelligence by Winners, Losers and Observers (Two Observers' ratings were combined in the figure. The vertical bars show standard deviations. The two downward arrows show the Modesty Effect; Winners and Losers rated themselves lower than the others did.)

$\left(F_{(2,160)}=52.67, p<.01\right)$. It was rather hard to interpret the result that overall scores were higher in the Strangers condition than in the Friends condition $\left(F_{(1,80)}=5.10, p<.05\right)$. This unexpected difference seems little-related to the objectives of the present study, so it was left open here. The main effect of Rater roles (Winners-Losers-Observers-Observers) was not significant, but the interaction between Rater roles and Rated roles was significant. The participants rated similarly irrespective of their roles in the social comparison setting $\left(F_{(3,80)}=.79, n s\right)$. On the other hand, they rated differently the winners' and losers' intelligence depending on their roles $\left(F_{(6,160)}=7.41, p<.01\right)$. Post hoc comparisons using the LSD method revealed that both Winners and Losers rated themselves lower than did the others $(p<.05)$. The other interactions were not significant.

\section{Modesty Effect in Japanese}

In Alicke et al. (1997), losers scored their own intelligence level similar to the ratings of others while they rated the intelligence of their contestants much higher than the others did (the Genius Effect). In the same vein, the winners in Lassiter et al. (2008) rated their opponents' ability lower than the observers did (the Idiot Effect). Mori and Mori (2011) found an opposite tendency in that both winners and losers rated themselves lower than the others did (the Modesty Effect) while they rated their opponents in a similar way as the others. The present finding replicated the results of Mori and Mori (2011) for both the Be-
tween-Friends and Between-Strangers conditions. The BetweenStrangers condition in the present study was virtually equivalent to the Alicke et al. and Lassiter et al. experiments, except for the cultural difference of the participants, Japanese in the present study and Americans in Alicke et al. and Lassiter et al. Therefore, it can be interpreted that the difference found in those studies derived from the cultural difference of the participants. Americans tended to attribute the outcomes of comparisons to the excessive ability or inability of their rivals, whereas Japanese would attribute them to their own ability or lack of it.

\section{Discussion}

\section{Why Japanese Are Modest}

Being modest is a useful tool for maintaining good social relations in any culture (Sedikides, Gregg, \& Hart, 2007). Then, why did only the Japanese participants in the present study show the Modesty Effect, but not those in Alicke et al. (1997) or Lassiter et al.? One possible explanation is that the American participants were asked to evaluate the ability of their opponents (confederates) who were unfamiliar to them. Since they knew little about their opponents, they put more weight on their own abilities as a reference criterion. If they were outperformed, it would be natural to assume that their opponents' ability was superior to their own. When they outperformed their opponents, the losers' ability could be assumed to be inferior to theirs. On the other hand, in the present study, the opponents were not unfamiliar strangers even in the Between-strangers condition. They came from the same campus. Students in Japanese universities are filtered and layered by a uniform entrance examination system conducted nation-wide. Therefore, the participants in the present experiments should have had a good estimate of the intellectual level of their counterparts even if they were strangers to each other. Consequently, they could not attribute the outcome to their opponent being a genius or an idiot.

It should be noted that the participants in the present study might have answered pretending to be modest. They might have evaluated the losers as much less competent and the winners as far superior as those participants in Alicke et al. and Lessiter et al., but they might have hidden their honest evaluations to enhance their reputation among their friends and others. Yamaguchi, Greenwald, Banaji, Murakami, Chen, Shiomura, Kobayashi, and Cai (2007) revealed that cultural differences in selfesteem between West and East were not observed in implicit measures, such as Implicit Association Tests (IAT; Greenwald, McGhee, \& Schwartz, 1998). Students in the East not only answered modestly in questionnaires, but they showed a similar response pattern in the IAT, in which their hidden attitudes were revealed. It would be desirable to include these implicit measures in social comparison studies in the future. Mori, Uchida, and Imada (2008) developed a paper-and-pencil version of the IAT. This would be suitable because it could be easily used with a conventional questionnaire.

\section{Conclusion}

American participants evaluated their opponents' ability higher to protect their own self-esteem when they were outperformed (Genius Effect) as demonstrated in Alicke, et al. (1997). Americans also showed a similar tendency when they outperformed their opponents, rating them even lower than them- 
selves (Idiot Effect, Lassiter et al., 2008). However, Japanese participants showed different rating tendencies. Japanese losers did not evaluate their opponents' ability higher than did the observers, irrespective of whether they were friends or strangers to each other. Instead, they tended to evaluate their own ability lower than the others did. Even when they outperformed their opponents, they evaluated themselves lower than the observers did. Further cross-cultural research is needed to investtigate these differences with participants from different cultural backgrounds other than American and Japanese. It might also be desirable to conduct a social comparison experiment among friends, rather than strangers, with American participants. The new experimental procedure used in the present study, in which winners and losers were created without the use of confederates, could be used for experiments in a variety of social comparison studies. It is also recommended that such studies utilize implicit measures, such as the IAT, as well as conventional explicit rating measures.

\section{Acknowledgements}

This research was supported by Grants-in-Aid from the Japanese Ministry of Education, Culture, Sports, Science, and Technology (Grant No.19330157 and 21012002) to KM. It was approved by the ethical committee of Tokyo University of Agriculture and Technology in 2009. The authors are indebted to Rebecca Ann Marck for her superb work in editing the English manuscript.

\section{REFERENCES}

Alicke, M. D., LoSchiavo, F. M., Zerbst, J., \& Zhang, S. (1997). The person who outperforms me is a genius: Maintaining perceived competence in upward social comparison. Journal of Personality and So- cial Psychology, 73, 781-789.

http://dx.doi.org/10.1037/0022-3514.73.4.781

Greenwald, A. G., McGhee, D. E., \& Schwartz, J. K. L. (1998). Measuring individual differences in implicit cognition: The Implicit Association Test. Journal of Personality and Social Psychology, 74, 14641480. http://dx.doi.org/10.1037/0022-3514.74.6.1464

Lassiter, G. D., Clark, J. K., Munhall, P. J., \& Lindberg, M. J. (2008). And I thought I was bad! The Idiot Effect in social judgment. Social Cognition, 26, 347-356. http://dx.doi.org/10.1521/soco.2008.26.3.347

Lassiter, G. D., \& Munhall, P. J. (2001). The Genius Effect: Evidence for a non-motivational interpretation. Journal of Experimental Social Psychology, 37, 349-355. http://dx.doi.org/10.1006/jesp.2000.1463

Mori, K. (2007). Projecting two words with one machine: A method for presenting two different visual stimuli using just one projector without viewers' noticing the duality. Behavior Research Methods, 39, 811-815. http://dx.doi.org/10.3758/BF03192973

Mori, K., \& Mori, H. (2011). No confederates needed: Social comparison without collaboration. Social Behavior and Personality, 39, 543552. http://dx.doi.org/10.2224/sbp.2011.39.4.543

Mori, K., Uchida, A., \& Imada, R. (2008). A paper-format group performance test for measuring the implicit association of target concepts. Behavior Research Methods, 40, 546-555.

http://dx.doi.org/10.3758/BRM.40.2.546

Sedikides, C., Gregg, A. P., \& Hart, C. M. (2007). The importance of being modest. In C. Sedikides, \& S. Spencer (Eds.), The self: Frontiers in social psychology (pp. 163-184). New York, NY: Psychology Press.

Yamaguchi, S., Greenwald, A. G., Banaji, M. R., Murakami, F., Chen, D., Shiomura, K., Kobayashi, C., \& Cai, H. (2007). Apparent universality of positive implicit self-esteem. Psychological Science, 18, 498-500. http://dx.doi.org/10.1111/j.1467-9280.2007.01928.x 